\title{
On the Asymptotic Behaviors of Transition Probability Densities of One-Dimensional Diffusion Processes
}

\author{
By \\ Matsuyo TomisAKI*
}

\section{§1. Introduction}

Let $\mathbf{X}^{*}=\left[X^{*}(t), P_{x}^{*}, x \in Q\right]$ be a one-dimensional diffusion processes on an interval $Q\left(\subseteq R^{1}\right)$ and $p(t, x, y)$ be its transition probability density with respect to the speed measure $m(d x)$. We are interested in the problem of describing the asymptotic behavior of $p(t, a, a)$ as $t \rightarrow 0[\infty]$ in terms of the speed measure $m$. Concerning this problem, there have been several works when $a \in Q$ is the regular left end point except a trap: By completing I.S. Kac's result [3], Kasahara [4] showed that $p(t, a, a)$ varies regularly in $t$ if and only if $m[a, x)$ does so in $x$. I. S. Kac [2] discussed the condition of the convergence of integrals related to the spectral function corresponding to the measure $m(d x)$ and this result gives conditions for the convergence of the integrals $\int_{0+} \varphi(t) p(t$, $a, a) d t$ and $\int^{\infty} \varphi(t) p(t, a, a) d t$ in terms of the measure $m(d x)$ for some class of positive nonincreasing functions $\varphi(t)$.

In this paper we remove the restriction that $a$ is the left end point and obtain the following results for the general case of an interior point or a regular end point. Our main results are following: First, we obtain some inequalities estimating the growth order of the function $p(t, a, a)$ when $t \rightarrow 0$ or $+\infty$ using some nonincreasing functions $F_{a}(t)$ defined in terms of the speed measure $m$. The proof of these inequalities is based on some inequalities similar to that of I. S. Kac [3] concerning

Communicated by K. Itô, August 17, 1976.

* Department of Mathematics, Saga University, Saga 840, Japan. 
$G(\alpha, a, a)=\int_{0}^{\infty} e^{-\alpha t} p(t, a, a) d t$ which we will prove by a probabilistic method. Second, using these inequalities we obtain necessary and sufficient conditions, in terms of the speed measure $m$, for the convergence of the integrals $\int_{0+} \varphi(t) p(t, a, a) d t$ and $\int^{\infty} \varphi(t) p(t, a, a) d t$ for any positive and nonincreasing function $\varphi(t)$.

Our results will be applied to obtain, for a two-dimensional diffusion process which is given as a direct product of one-dimensional diffusion processes, necessary and sufficient conditions for possibility of hitting a given point and for recurrence or transience of the diffusion.

Finally the author would like to thank Professor S. Watanabe, Professor N. Ikeda and Professor Y. Ogura for their several valuable suggestions.

\section{§2. Main Theorems}

Let $l_{1}\left[l_{2}\right]$ be the left [right] end point $Q$. Let $m(d x)$ and $k(d x)$ be nonnegative Borel measures on $\left(l_{1}, l_{2}\right)$, which are finite on each closed subinterval of $\left(l_{1}, l_{2}\right)$, where $m(U)>0$ for any open set $U(\neq \phi)$. Denote the generator of $\mathbf{X}^{\circ}$ by $\mathbf{5}^{\circ}$ :

$$
\left(5^{\circ} u\right)(x)=\left\{u^{+}(d x)-u(x) k(d x)\right\} / m(d x),{ }^{(1)} \quad x \in Q .
$$

If $l_{i} \in Q$, then the boundary condition is given by

$\left(l_{i}-1\right) \quad(-1)^{i} u^{i}\left(l_{i}\right)+k_{i} u\left(l_{i}\right)+m_{i}\left(5^{\cdot} u\right)\left(l_{i}\right)=0$,

or

$\left(l_{i}-2\right) \quad((5) u)\left(l_{i}\right)=-\kappa_{i} u\left(l_{i}\right)$,

where $0 \leqq k_{i}, m_{i}, \kappa_{i}<\infty$ and $u^{1}\left(l_{1}\right) \equiv u^{+}\left(l_{1}+\right), u^{2}\left(l_{2}\right) \equiv u^{-}\left(l_{2}-\right) .^{(2)} \quad$ If $l_{i} \in Q$, we extend $m(d x)$ and $k(d x)$ on $Q$ so that

$$
\begin{array}{ll}
m\left(\left\{l_{i}\right\}\right)=m_{i}, k\left(\left\{l_{i}\right\}\right)=k_{i} & \text { if } \quad\left(l_{i}-1\right), \\
m\left(\left\{l_{i}\right\}\right)=k\left(\left\{l_{i}\right\}\right)=0, & \text { if }\left(l_{i}-2\right) .
\end{array}
$$

(1) (2) $u^{+}(x)=\lim _{y \downarrow x} \frac{u(y)-u(x)}{s(y)-s(x)}, u^{-}(x)=\lim _{y \uparrow x} \frac{u(x)-u(y)}{s(x)-s(y)}$,

where $s(x)$ is the scale, i.e. a continuous increasing function on $\left(l_{1}, l_{2}\right)$. 
Let $Q^{r}$ be the set of all points $a \in Q$ which satisfy either of the following conditions:

(i) $l_{1}<a<l_{2}$,

(ii) $a=l_{i}$ with $\left(l_{i}-1\right)$ and $s\left(l_{i}\right)$ is bounded, where $s\left(l_{1}\right) \equiv s\left(l_{1}+\right)$ and $s\left(l_{2}\right) \equiv s\left(l_{2}-\right)$.

Let us define the following functions:

$$
\begin{aligned}
& \bar{U}_{a}(x)=\int_{0}^{x} m \circ t[s(a), s(a)+y) d y, \\
& U_{a}(x)=\int_{0}^{x} m \circ t(s(a)-y, s(a)] d y, \\
& U_{a}(x)=\int_{0}^{x} m \circ t(s(a)-y, s(a)+y) d y, \\
& \bar{V}_{a}(x)=x m \circ t[s(a), s(a)+x), \\
& \underline{V}_{a}(x)=x m \circ t(s(a)-x, s(a)], \\
& V_{a}(x)=x m \circ t(s(a)-x, s(a)+x),
\end{aligned}
$$

where $t(x)$ is the inverse function of $s(x)$ and $m \circ t(a, b]=m(t(a), t(b)]$. Let $\bar{\Phi}_{a}(x)\left[\bar{\Psi}_{a}(x)\right], \underline{\Phi}_{a}(x)\left[\underline{\Psi}_{a}(x)\right]$ and $\Phi_{a}(x)\left[\Psi_{a}(x)\right]$ be the inverse functions of $x \mapsto \bar{U}_{a}(x)\left[\bar{V}_{a}(x)\right], x \mapsto \underline{U}_{a}(x)\left[\underline{V}_{a}(x)\right]$ and $x \mapsto U_{a}(x)\left[V_{a}(x)\right]$ respectively. Let us put $P_{a}(t)=\int_{0}^{t} p(s, a, a) d s$.

Through this paper we shall introduce the following notation: we write

$$
a(t) \asymp b(t) \text { as } \quad t \downarrow 0[t \uparrow \infty]
$$

if and only if

$$
0<\lim _{\substack{t \downarrow 0 \\ t \uparrow 0 \\ t \uparrow \uparrow \infty]}} \frac{a(t)}{b(t)} \leqq \varlimsup_{\substack{t \downarrow 0 \\[t \uparrow \infty]}} \frac{a(t)}{b(t)}<\infty .
$$

First we study the asymptotic behavior of $P_{a}(t)$ as $t \downarrow 0$.

Theorem 2.1. For every $a \in Q^{r}$,

$$
P_{a}(t) \asymp F_{a}(t) \quad \text { as } \quad t \downarrow 0 \text {, }
$$


where

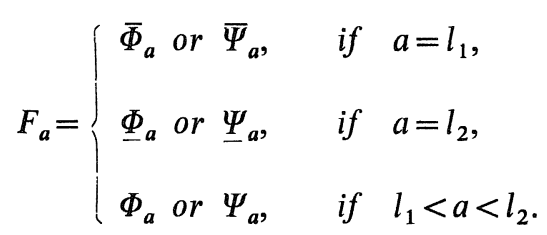

Remark. In particular we see $\bar{\Phi}_{a}(t) \asymp \bar{\Psi}_{a}(t), \underline{\Phi}_{a}(t) \asymp \underline{\Psi}_{a}(t)$ and $\Phi_{a}(t)$ $\asymp \Psi_{a}(t)$ as $t \downarrow 0$ which can be verified directly.

Next we study the case $t \uparrow \infty$. We are interested only in the case $\lim _{t \uparrow \infty} P_{a}(t)=\infty\left(a \in Q^{r}\right)$, which holds if and only if the following conditions are satisfied:

$(\mathrm{P}-1) \quad k(Q)=0$,

(P-2) if $s\left(l_{1}\right)>-\infty$, then $m\left(l_{1}, l_{0}\right]<\infty$ and $l_{1} \in Q$ with $\left(l_{1}-1\right),{ }^{(3)}$

(P-3) if $s\left(l_{2}\right)<\infty$, then $m\left[l_{0}, l_{2}\right)<\infty$ and $l_{2} \in Q$ with $\left(l_{2}-1\right)_{,}{ }^{(4)}$

where $l_{0}$ is any fixed point in $\left(l_{1}, l_{2}\right)$. If, besides $(\mathrm{P}-1),(\mathrm{P}-2)$ and (P-3), $m(Q)<\infty$ is satisfied, then we have $\lim _{t \uparrow \infty} P_{a}(t) / t=1 / m(Q)$. Therefore we consider the remaining case $m(Q)=\infty$. ${ }^{t}$ We may suppose $m\left[l_{0}, l_{2}\right)=$ $\infty$ without loss of generality.

Theorem 2.2. Assume (P-1), $(\mathrm{P}-2), s\left(l_{2}\right)=\infty$ and $m\left[l_{0}, l_{2}\right)=\infty$. Then for every $a \in Q^{r}$,

$$
P_{a}(t) \asymp H_{a_{0}}(t) \quad \text { as } \quad t \uparrow \infty,
$$

where $a_{0}$ is any fixed point in $\left(l_{1}, l_{2}\right)$ and

$$
H_{a}=\left\{\begin{array}{lll}
\bar{\Phi}_{a} \text { or } \bar{\Psi}_{a}, & \text { if } & m\left(l_{1}, l_{0}\right]<\infty, \\
\Phi_{a} \text { or } \Psi_{a}, & \text { if } & m\left(l_{1}, l_{0}\right]=\infty
\end{array}\right.
$$

These asymptotic behaviors of $P_{a}(t)$ are equivalent to those of the Green function. Let $G(\alpha, x, y)$ be the Green function of $\mathbf{X}^{*}$, i.e. $G(\alpha$,

(3), (4) If (P-1) and (P-2) [(P-3)] are satisfied, then $\left(l_{i}-1\right)$ means $(-1)^{i} u^{i}\left(l_{i}\right)+$ $m^{i}\left(\Im^{\circ} u\right)\left(l_{i}\right)=0$. 
$x, y)=\int_{0}^{\infty} e^{-\alpha . t} p(t, x, y) d t$. Then Theorem $2.1[2.2]$ is equivalent to the following Theorem 2.1' [2.2'], which can be proved by using [5; Lemma 2.1] noting $G(\alpha, a, a)=\int_{0}^{\infty} e^{-\alpha t} P_{a}(d t)$ and $P_{a}(t)$ is positive, increasing and concave.

Theorem 2.1'. For every $a \in Q^{r}$,

$$
G(\alpha, a, a) \asymp F_{a}(1 / \alpha) \quad \text { as } \quad \alpha \uparrow \infty \text {. }
$$

Theorem 2.2'. Assume (P-1), $(\mathrm{P}-2), s\left(l_{2}\right)=\infty$ and $m\left[l_{0}, l_{2}\right)=\infty$. Then for every $a \in Q^{r}$,

$$
G(\alpha, a, a) \asymp H_{a_{0}}(1 / \alpha) \quad \text { as } \quad \alpha \downarrow 0,
$$

where $a_{0}$ is any fixed point in $\left(l_{1}, l_{2}\right)$.

Since $\lim _{\alpha \downarrow 0} G(\alpha, a, b) / G(\alpha, c, d)=1\left(a, b, c, d \in Q^{r}\right)$ under the assumption of Theorem 2.2 or Theorem 2.2', Theorem 2.2 and Theorem 2.2' may be summarized in the following table:

\begin{tabular}{|c|c|c|c|}
\hline$k(Q)=0$ & $s\left(l_{2}\right)$ & $<\infty, l_{2} \in Q,\left(l_{2}-1\right)$ & $=\infty$ \\
\hline$s\left(l_{1}\right)$ & $m\left[l_{0}\right.$ & $<\infty$ & $=\infty$ \\
\hline $\begin{array}{l}>-\infty \\
l_{1} \in Q \\
\left(l_{1}-1\right)\end{array}$ & $<\infty$ & $\begin{array}{l}\lim _{\alpha \downarrow 0} \alpha G(\alpha, a, b)=1 / m(Q) \\
\lim _{t \uparrow \infty} P_{a}(t) / t=1 / m(Q)\end{array}$ & $\begin{array}{r}G(\alpha, a, b) \asymp \bar{\Phi}_{c}(1 / \alpha) \text { or } \\
\bar{\Psi}_{c}(1 / \alpha) \text { as } \alpha \downarrow 0 \\
P_{a}(t) \asymp \bar{\Phi}_{c}(t) \text { or } \bar{\Psi}_{c}(t) \\
\text { as } t \uparrow \infty\end{array}$ \\
\hline$=\infty$ & $=\infty$ & $\begin{array}{r}G(\alpha, a, b) \asymp \underline{\Phi}_{c}(1 / \alpha) \text { or } \\
\underline{\Psi}_{c}(1 / \alpha) \text { as } \alpha \downarrow 0 \\
P_{a}(t) \asymp \underline{\Phi}_{c}(t) \text { or } \underline{\Psi}_{c}(t) \\
\text { as } t \uparrow \infty\end{array}$ & $\begin{array}{r}G(\alpha, a, b) \asymp \Phi_{c}(1 / \alpha) \text { or } \\
\Psi_{c}(1 / \alpha) \text { as } \alpha \downarrow 0 \\
P_{a}(t) \asymp \Phi_{c}(t) \text { or } \Psi_{c}(t) \\
\text { as } t \uparrow \infty\end{array}$ \\
\hline
\end{tabular}

Table 2.1

where $a, b \in Q^{r}$ and $c\left(\neq l_{i}\right)$ is any fixed point in $Q^{r}$. 


\section{§3. Proofs of the Main Theorems}

First, we obtain some inequalities on $G(\alpha, x, y)$ to prove Theorem $2.1^{\prime}$ and Theorem $2.2^{\prime}$. For this we introduce the conservative diffusion $\boldsymbol{X}=\left[X(t), P_{x}, x \in Q\right]$ with the same $m(d x)$ and $s(x)$ as $\boldsymbol{X}^{*}$. In other words, its generator $(5)$ is given by $(\mathfrak{F} u)(x)=u^{+}(d x) / m(d x)$ and if $l_{i} \in Q$, the boundary condition is

$$
\begin{array}{r}
(-1)^{i} u^{i}\left(l_{i}\right)+m_{i}(\mathfrak{5} u)\left(l_{i}\right)=0, \quad \text { in case }\left(l_{i}-1\right), \\
(\mathfrak{5} u)\left(l_{i}\right)=0, \quad \text { in case }\left(l_{i}-2\right) .
\end{array}
$$

Denoting the local time at $x$ by $\mathrm{t}(t, x)$ (i.e. $\int_{A} \mathrm{t}(t, x) m(d x)=\int_{0}^{t} I_{A}(X(s)) d s$, $A \in \mathfrak{B}(Q)$ ), we have

$$
G(\alpha, a, b)=E_{a}\left[\int_{0}^{\infty} e^{\mathfrak{t}(t, \alpha) \mathrm{t}(d t, b)],{ }^{(5)}}\right.
$$

where $\mathfrak{f}(t, \alpha)=-\alpha t-\int_{Q} \mathfrak{t}(t, x) k(d x)-\mathfrak{f}_{1}(t)-\mathfrak{f}_{2}(t)$,

$$
\mathfrak{f}_{i}(t)=\left\{\begin{array}{lr}
\kappa_{i} \times \text { Lebesgue measure of }\left\{s ; X(s)=l_{i}, s \leqq t\right\} \\
0, \quad \text { otherwise. }
\end{array}\right.
$$

Define $\sigma_{x}$ by

$$
\sigma_{x}=\min \{t ; X(t)=x, t \geqq 0\} \quad(x \in Q) .
$$

It follows from the strict Markov property that

$$
\begin{aligned}
& G(\alpha, a, a)=\frac{h_{\xi \eta}(a, a, \alpha)}{1-g_{\xi \eta}(a, \alpha) f(\xi, a, \alpha)-g_{\eta \xi}(a, \alpha) f(\eta, a, \alpha)}, \\
& \text { for } \quad a, \xi, \eta \in Q^{r} \quad(\xi<a<\eta),
\end{aligned}
$$

and in particular if $m\left(l_{1}, l_{0}\right]<\infty$ and $(\mathrm{P}-2)$ is satisfied, then

(5) See $[1 ; \S 5.6]$. 


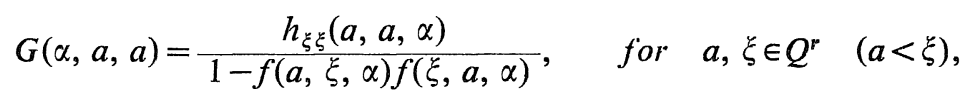

where

$$
\begin{aligned}
& f(x, y, \alpha)=E_{x}\left[e^{\mathfrak{\ell}\left(\sigma_{y}, \alpha\right)}\right], \\
& g_{\xi_{\eta}}(x, \alpha)=E_{x}\left[e^{\ell\left(\sigma_{\xi}, \alpha\right)} ; \sigma_{\xi}<\sigma_{\eta}\right], \\
& h_{\xi \eta}(x, y, \alpha)=E_{x}\left[\int_{0}^{\sigma_{\xi} \wedge \sigma_{\eta}} e^{f(t, \alpha)} \mathrm{t}(d t, y)\right] .
\end{aligned}
$$

Assume that points $x, y, \xi, \eta$ in $f, g, h$ are in $Q^{r}$. For simplicity we write $i_{\alpha}(d x)=\alpha m(d x)+k(d x)$ and

$$
\begin{aligned}
& \bar{I}_{a}(x, \alpha)=\int_{a}^{x} i_{\alpha}[a, y) s(d y), \\
& \underline{I}_{a}(x, \alpha)=\int_{x}^{a} i_{\alpha}(y, a) s(d y), \\
& \bar{J}_{a}(x, \alpha)=(s(x)-s(a)) i_{\alpha}[a, x), \\
& J_{a}(x, \alpha)=(s(a)-s(x)) i_{\alpha}(x, a) .
\end{aligned}
$$

We show the following inequalities, which are analogous to I.S. Kac's onc [3; Lemma 2.7].

Lemma 3.1. Let $a$ be any' point of $Q^{r}\left(a<l_{2}\right)$. Let $\bar{K}_{a}\left[\underset{\sim}{K_{a}}\right]$ be

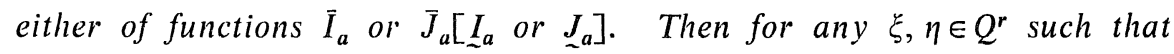
$\check{\zeta}<a<\eta$,

$$
\begin{aligned}
& \varepsilon \delta /\left\{\varepsilon+\delta+\varepsilon \bar{K}_{a}(\eta, \alpha)+\delta \underset{\sim}{K_{a}}(\xi, \alpha)\right\} \\
& \quad \leqq G(\alpha, a, a) \\
& \quad \leqq \varepsilon \delta\left\{\varepsilon+\delta+\varepsilon \bar{K}_{a}(\eta, \alpha)+\delta{\underset{\sim}{K}}_{a}(\xi, \alpha)\right\} /\left\{\varepsilon^{2} \bar{K}_{a}(\eta, \alpha)+\delta^{2}{\underset{\sim}{a}}_{a}(\xi, \alpha)\right\},
\end{aligned}
$$

where $\varepsilon=s(a)-s(\xi)$ and $\delta=s(\eta)-s(a)$. In particular, if $m\left(l_{1}, l_{0}\right]<\infty$ and $(\mathrm{P}-2)$ is satisfied, then for every $\xi \in Q^{r}$ such that $a<\xi$,

$$
\delta /\left\{1+\delta W(a, \alpha)+\bar{K}_{a}(\xi, \alpha)\right\} \leqq G(\alpha, a, a) \leqq \delta\left\{1+1 / \bar{K}_{a}(\zeta, \alpha)\right\},
$$


where $\delta=s(\xi)-s(a)$ and

$$
W(a, \alpha)= \begin{cases}\int_{\left[l_{1}, a\right) \cap Q} f(x, a, \alpha) i_{\alpha}(d x), & \text { if } a>l_{1}, \\ 0, & \text { if } a=l_{1} .\end{cases}
$$

Proof. First we show (3.3). Since $h_{\xi \eta}(x, y, \alpha)$ satisfies

$$
h_{\xi \eta}(x, y, \alpha)=e_{\xi \eta}(x, y)-\int_{\xi}^{\eta} e_{\xi \eta}(x, z) h_{\xi \eta}(z, y, \alpha) i_{\alpha}(d z),{ }^{(6)}
$$

for $x, y \in(\xi, \eta)$, where $\quad e_{\xi \eta}(x, y)=e_{\xi \eta}(y, x)=\{s(x)-s(\xi)\}\{s(\eta)-s(y)\} /\{s(\eta)-$ $s(\xi)\}(\xi \leqq x \leqq y \leqq \eta)$, by using $h_{\xi \eta}(x, y, \alpha) \leqq h_{\xi \eta}(y, y, \alpha)$ we have

$$
\begin{aligned}
h_{\xi \eta}(a, a, \alpha) & \geqq \varepsilon \delta /\left\{\varepsilon+\delta+\varepsilon \bar{I}_{a}(\eta, \alpha)+\delta \underline{I}_{a}(\xi, \alpha)\right\} \\
& \geqq \varepsilon \delta /\left\{\varepsilon+\delta+\varepsilon \bar{J}_{a}(\eta, \alpha)+\delta J_{\sim}(\xi, \alpha)\right\} .
\end{aligned}
$$

Because of $G(\alpha, a, a) \geqq h_{\xi \eta}(a, a, \alpha)$ and (3.1), we obtain the lower estimate of $G(\alpha, a, a)$.

Since $f(\cdot, \xi, \alpha)$ is a solution of $(5 u=\alpha u$ and satisfies

$$
(-1)^{i} f^{i}\left(l_{i}, \xi, \alpha\right)+\left(k_{i}+\alpha m_{i}\right) f_{i}\left(l_{i}, \xi, \alpha\right)=0, \quad \text { if } \quad l_{i} \in Q \text { and }\left(l_{i}-1\right)
$$

we have the estimate

$$
f(a, \xi, \alpha) \leqq\left\{\begin{array}{lll}
\left(1+\bar{I}_{a}(\xi, \alpha)\right)^{-1}, & \text { if } & a<\xi, \\
\left(1+\underline{I}_{a}(\xi, \alpha)\right)^{-1}, & \text { if } & a>\xi .
\end{array}\right.
$$

On the other hand, it is easy to see that $g_{\xi_{\eta}}(x, \alpha)$ is nonincreasing whereas $g_{\eta \xi}(x, \alpha)$ is nondecreasing and that they satisfy

$$
\begin{aligned}
& g_{\xi \eta}(x, \alpha)=\frac{s(\eta)-s(x)}{s(\eta)-s(\xi)}-\int_{\xi}^{\eta} e_{\xi \eta}(x, y) g_{\xi \eta}(y, \alpha) i_{\alpha}(d y) \\
& g_{\eta \xi}(x, \alpha)=\frac{s(x)-s(\xi)}{s(\eta)-s(\xi)}-\int_{\xi}^{\eta} e_{\xi \eta}(x, y) g_{\eta \xi}(y, \alpha) i_{\alpha}(d y),{ }^{(7)}
\end{aligned}
$$

for $x \in[\xi, \eta]$, and hence

(6) The proof is similar to $[1 ; \S 5.6(16)]$.

(7) See $[1 ; \S 5.6(12)]$. 


$$
\begin{aligned}
& g_{\xi \eta}(a, \alpha) \leqq\left\{\frac{\varepsilon+\delta}{\delta}+\underline{I}_{a}(\zeta, \alpha)\right\}^{-1}, \\
& g_{\eta \xi}(a, \alpha) \leqq\left\{\frac{\varepsilon+\delta}{\varepsilon}+\bar{I}_{a}(\eta, \alpha)\right\}^{-1} .
\end{aligned}
$$

We combine (3.5) and (3.6) to obtain

$$
\begin{aligned}
f(\xi, a, \alpha) g_{\xi \eta}(a, \alpha) & \leqq\left\{\frac{\varepsilon+\delta}{\delta}+\underline{J}_{a}(\xi, \alpha)\right\}^{-1} \\
& \leqq\left\{\frac{\varepsilon+\delta}{\delta}+\underline{I}_{a}(\xi, \alpha)\right\}^{-1} \\
f(\eta, a, \alpha) g_{\eta \xi}(a, \alpha) & \leqq\left\{\frac{\varepsilon+\delta}{\varepsilon}+\bar{J}_{a}(\eta, \alpha)\right\}^{-1} \\
& \leqq\left\{\frac{\varepsilon+\delta}{\varepsilon}+\bar{I}_{a}(\eta, \alpha)\right\}^{-1} .
\end{aligned}
$$

Since $h_{\xi \eta}(a, a, \alpha) \leqq e_{\xi \eta}(a, a)=\varepsilon \delta /(\varepsilon+\delta)$, by (3.1) we have the upper estimate of $G(\alpha, a, a)$ in (3.3).

Next we show (3.4). Since $h_{\xi \xi}(x, y, \alpha)$ satisfies

$$
h_{\xi \xi}(x, y, \alpha)=e_{\xi}(x, y)-\int_{\left[l_{1}, \xi\right)} e_{\xi}(x, z) h_{\xi \xi}(z, y, \alpha) i_{\alpha}(d z),{ }^{(8)}
$$

for $\quad x, y<\xi$, where $e_{\xi}(x, y)=e_{\xi}(y, x)=s(\xi)-s(y)(x \leqq y \leqq \xi), \quad$ by $\quad$ using $h_{\xi \xi}(x, y, \alpha) \leqq h_{\xi \xi}(y, y, \alpha)$ we have

$$
\begin{aligned}
h_{\xi \xi}(a, a, \alpha) & \geqq \delta /\left\{1+\delta W(a, \alpha)+\bar{I}_{a}(\xi, \alpha)\right\} \\
& \geqq \delta /\left\{1+\delta W(a, \alpha)+\bar{J}_{a}(\xi, \alpha)\right\} .
\end{aligned}
$$

Therefore we obtain the lower estimate in (3.4) because $G(\alpha, a, a) \geqq h_{\xi \xi}(a$, $a, \alpha)$ and (3.2).

The upper estimate follows from (3.2), $h_{\xi \xi}(a, a, \alpha) \geqq e_{\xi}(a, a)=\delta$ and

$$
\begin{aligned}
f(a, \xi, \alpha) f(\xi, a, \alpha) & \leqq\left\{1+\bar{J}_{a}(\xi, a)\right\}^{-1} \\
& \leqq\left\{1+\bar{I}_{a}(\xi, a)\right\}^{-1}, \quad \text { if } a<\xi
\end{aligned}
$$

(8) The proof is similar to $[1 ; \S 5.6(16)]$. 
where we used (3.5). Thus we obtain the assertion of the lemma. Q.E.D.

We are ready to prove Theorem $2.1^{\prime}$ and Theorem $2.2^{\prime}$.

Proof of Theorem 2.1'. Suppose $l_{1}<a<l_{2}$. There is a $\delta>0$ such that $t(s(a)-\delta), t(s(a)+\delta) \in Q^{r}$. Taking $\xi=t(s(a)-\delta)$ and $\eta=t(s(a)+\delta)$ in (3.3), we have

$$
\begin{aligned}
\delta /\{2 & \left.+\bar{K}_{a}(t(s(a)+\delta), \alpha)+\underset{\sim}{K_{a}}(t(s(a)-\delta), \alpha)\right\} \\
& \leqq G(\alpha, a, a) \\
& \leqq \delta\left(1+2 / \alpha V_{a}(\delta)\right) \\
& \leqq \delta\left(1+2 / \alpha U_{a}(\delta)\right),
\end{aligned}
$$

where $U_{a}$ and $V_{a}$ are the functions defined in Section 2, and hence setting $\delta=F_{a}(1 / \alpha)$, we have

$$
\begin{aligned}
& F_{a}(1 / \alpha) /\left\{3+F_{a}(1 / \alpha) k \circ t\left(s(a)-F_{a}(1 / \alpha), s(a)+F_{a}(1 / \alpha)\right)\right\} \\
& \quad \leqq G(\alpha, a, a) \\
& \quad \leqq 3 F_{a}(1 / \alpha),
\end{aligned}
$$

for every $\alpha>0$ such that $t\left(s(a) \pm F_{a}(1 / \alpha)\right) \in Q^{r}$. Since $\lim _{x \downarrow 0} F_{a}(x)=0$, we have

$$
1 / 3 \leqq \lim _{\alpha \uparrow \infty} G(\alpha, a, a) / F_{a}(1 / \alpha) \leqq \varlimsup_{\alpha \uparrow \infty} G(\alpha, a, a) / F_{a}(1 / \alpha) \leqq 3
$$

By the same way in case $a=l_{i}$ we obtain

$$
1 / 2 \leqq \lim _{\alpha \uparrow \infty} G(\alpha, a, a) / F_{a}(1 / \alpha) \leqq \varlimsup_{\alpha \uparrow \infty} G(\alpha, a, a) / F_{a}(1 / \alpha) \leqq 2
$$

Thus the theorem is proved.

Q.E.D.

Proof of Theorem 2.2'. In case $m\left(l_{1}, l_{0}\right]=\infty$ the result is obvious from (3.7) and $\lim _{\alpha \downarrow 0} G(\alpha, a, a) / G(\alpha, b, b)=1\left(a, b \in Q^{r}\right)$. In case $m\left(l_{1}, l_{0}\right]$ $<\infty$ by the same method as in the proof of Theorem 2.1' it follows 
from (3.4) that

$$
H_{a}(1 / \alpha) /\left\{2+\alpha H_{a}(1 / \alpha) m\left(\left[l_{1}, a\right) \cap Q\right)\right\} \leqq G(\alpha, a, a) \leqq 2 H_{a}(1 / \alpha) .
$$

Put $M_{a}=\bar{U}_{a}$ or $\bar{V}_{a}$. Since $M_{a}(x) \geqq x m(a, A) / 2$ for all $x>B \equiv 2(s(A)-$ $s(a))$, where $A(>a)$ is any fixed number, we have $\alpha H_{a}(1 / \alpha) \leqq 2 / m(a, A)$ for all $\alpha \leqq 1 / M_{a}(B)$, so that

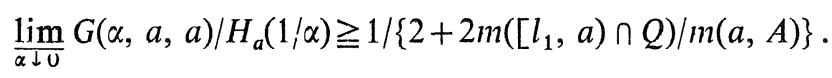

Since $m\left[l_{0}, l_{2}\right)=\infty$ and $A$ is arbitrary, letting $A \uparrow l_{2}$, we obtain

$$
1 / 2 \leqq \lim _{\alpha \downarrow 0} G(\alpha, a, a) / H_{a}(1 / \alpha) \leqq \varlimsup_{\alpha \downarrow 0} G(\alpha, a, a) / H_{a}(1 / \alpha) \leqq 2
$$

which completes the proof.

Q.E.D.

\section{§4. Integral Characteristics}

Let $\varphi$ be a positive nonincreasing function on $(0, \delta)[(\delta, \infty)]$ for some $\delta \in(0, \infty)$. By integration by parts and Theorem 2.1 [2.2] we have Theorem 4.1 [4.2] immediately.

Theorem 4.1. Fix any $a \in Q^{r}$. In order that the integral

$$
\int_{0}^{\delta} \varphi(t) p(t, a, a) d t
$$

converges, it is necessary and sufficient that the integral

$$
\int_{0}^{l} \varphi\left(u_{a}(x)\right) d x
$$

converges for some $l>0$, where

$$
u_{a}=\left\{\begin{array}{lll}
\bar{U}_{a} \text { or } \bar{V}_{a}, & \text { if } & a=l_{1}, \\
\underline{U}_{a} \text { or } \underline{V}_{a}, & \text { if } & a=l_{2}, \\
U_{a} \text { or } V_{a}, & \text { if } & l_{1}<a<l_{2} .
\end{array}\right.
$$

Theorem 4.2. Assume (P-1), ( $\mathrm{P}-2)$ and $(\mathrm{P}-3)$. In order that the 
integral

$$
\int_{\delta}^{\infty} \varphi(t) p(t, a, a) d t
$$

converges for some (and hence any) $a \in Q^{r}$, it is necessary and sufficient that the integral

$$
\int_{l}^{\infty} \varphi\left(v_{b}(x)\right) d x
$$

converges for some (and hence any) $b \in\left(l_{1}, l_{2}\right)$ and some $l>0$, where $v_{b}(x)$ is the function given in the following table:

Table 4.1

\begin{tabular}{l|c|c|c}
\hline$v_{b}(x)=$ & $s\left(l_{2}\right)$ & $<\infty, l \in Q,\left(l_{2}-1\right)$ & $=\infty$ \\
\hline$s\left(l_{1}\right)$ & $m\left[l_{0}, l_{2}\right)$ & $<\infty$ & $=\infty$ \\
& $m\left(l_{1}, l_{0}\right]$ & & \\
\hline$>-\infty$ & $x$ & $\bar{U}_{b}(x)$ or $\bar{V}_{b}(x)$ \\
$l_{1} \in Q$ & $<\infty$ & & \\
$\left(l_{1}-1\right)$ & & & \\
\hline & & $\underline{U}_{b}(x)$ or $\underline{V}_{b}(x)$ & $U_{b}(x)$ or $V_{b}(x)$ \\
\hline
\end{tabular}

\section{§5. Applications to Two-Dimensional Direct}

Product Diffusion Processes

Let $\boldsymbol{X}^{i}=\left[X^{i}(t), P_{x}^{i}, x \in Q^{i}\right](i=1,2)$ be a conservative one-dimensional diffusion process with the generator $\left(\mathfrak{5}^{i} u\right)(x)=u^{i+}(d x) / m^{i}(d x)$, where $u^{i+}(x)$ denotes the right derivative by $s^{i}(x)$. Assume that $\boldsymbol{X}^{i}$ satisfies the conditions $(\mathrm{P}-1),(\mathrm{P}-2)$ and $(\mathrm{P}-3)$ for each $i$. We define a twodimensional diffusion process $X$ on $Q=Q^{1} \times Q^{2}$ by $\mathbb{X}=\left[X(t)=\left(X^{1}(t)\right.\right.$, $\left.\left.X^{2}(t)\right), P_{x}=P_{x^{1}}^{1} \times P_{x^{2}}^{2}, x=\left(x^{1}, x^{2}\right) \in Q\right]$. Let $G(\alpha, x, y)$ be its Green function. On the possibility of hitting a single point $a\left(\in Q^{r} \equiv Q^{1 r} \times Q^{2 r}\right)$ 
for the sample path of $\boldsymbol{X}$, it is well known that $P_{x}\left(\sigma_{a}<\infty\right)>0\left(x \in Q^{r}\right)$ if and only if $G(\alpha, a, a)<\infty$. Also it is well known that the process is recurrent if and only if $\lim _{\alpha \downarrow 0} G(\alpha, a, b)=\infty\left(a, b \in Q^{r}\right)$. Combining these, we see that $P_{x}\left(\sigma_{a}<\infty\right)=1\left(x \in Q^{r}\right)$ if and only if $G(\alpha, a, a)<\infty$ and $\lim _{\alpha \downarrow 0} G(\alpha, a, a)=\infty$. Thus denoting $\bar{\Phi}_{a}^{i}(x)$ etc. by the inverse function of $\quad x \mapsto \bar{U}_{a}^{i}(x) \equiv \int_{0}^{x} m^{i} \circ t^{i}\left[s^{i}(a), s^{i}(a)+y\right) d y \quad$ etc., we have the following results from Theorem 4.1. and Theorem 4.2.

Theorem 5.1. Fix any point $a=\left(a^{1}, a^{2}\right) \in Q^{r}$. In order to $P_{x}\left(\sigma_{a}\right.$ $<\infty)>0$ for all $x \in Q^{r}$, it is necessary and sufficient that the integral

$$
\int_{0}^{\delta}\left\{\mu_{a^{1}}^{1}(x) \mu_{a^{2}}^{2}(x)\right\}^{-1} d x
$$

converges for some $\delta>0$, where

$$
\begin{aligned}
& \mu_{a^{i}}^{i}(x)= \begin{cases}m^{i} \circ t^{i}\left[s^{i}\left(a^{i}\right), s^{i}\left(a^{i}\right)+\bar{\Phi}_{a^{i}}^{i}(x)\right), & \text { if } \quad a^{i}=l_{1}^{i}, \\
m^{i} \circ t^{i}\left(s^{i}\left(a^{i}\right)-\Phi_{a^{i}}^{i}(x), s^{i}\left(a^{i}\right)\right], \quad \text { if } \quad a^{i}=l_{2}^{i}, \\
m^{i} \circ t^{i}\left(s^{i}\left(a^{i}\right)-\Phi_{a^{i}}^{i}(x), s^{i}\left(a^{i}\right)+\Phi_{a^{i}}^{i}(x)\right),\end{cases} \\
& \text { if } l_{1}^{i}<a^{i}<l_{2}^{i} .
\end{aligned}
$$

Theorem 5.2. In order that $\boldsymbol{X}$ is recurrent it is necessary and sufficient that the integral

$$
\int_{l}^{\infty}\left\{v_{a^{1}}^{1}(x) v_{a^{2}}^{2}(x)\right\}^{-1} d x
$$

diverges for some $l>0$ and some (and hence all) $a=\left(a^{1}, a^{2}\right) \in Q^{r}$, where $v_{a^{i}}^{i}(x)$ is the function given in Table 5.1.

Theorem 5.3. Fix any point $a=\left(a^{1}, a^{2}\right) \in Q^{r}$. In order to $P_{x}\left(\sigma_{a}\right.$ $<\infty)=1$ for all $x \in Q^{r}$, it is necessary and sufficient that the integral (5.1) converges for some $\delta>0$ and the integral (5.2) diverges for some $l>0$.

As an immediate result of Theorem 5.1 we obtain the following: 
Corollary 5.4. Two-dimensional Lebesgue measure of $\left\{a \in Q^{r}\right.$;

Table 5.1

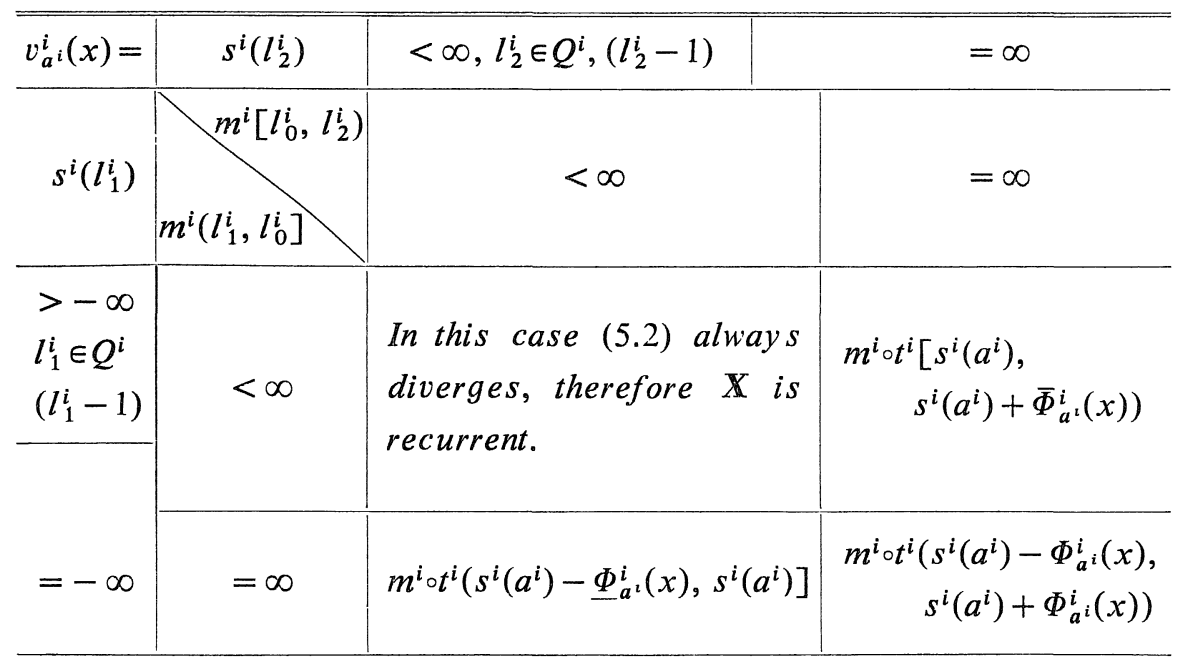

Table $\mathbf{5 . 2}$

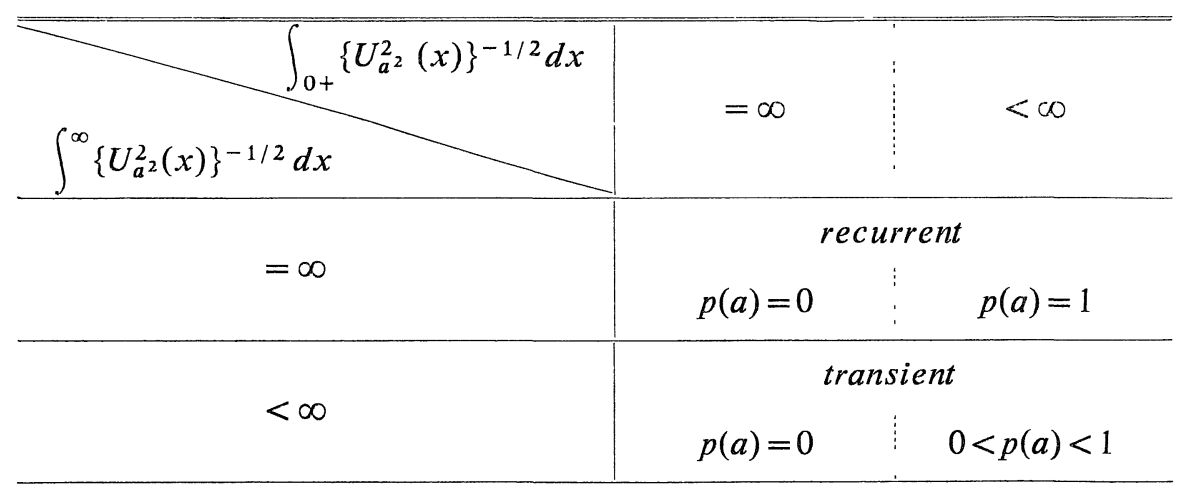

Table 5.3

\begin{tabular}{c|c|c|c}
\hline \hline$\gamma$ & $-1<\gamma<0$ & $\gamma=0$ & $\gamma>0$ \\
\hline $\mathbf{X}$ & \multicolumn{2}{c}{ recurrent } & transient \\
\hline$a \in A^{\circ}$ & $p(a)=1$ & $p(a)=0$ \\
\hline$a \notin A^{\circ}$ & \multicolumn{2}{|c}{$p(a)=0$} \\
\hline
\end{tabular}


$P_{x}\left(\sigma_{a}<\infty\right)>0$ for every $\left.x \in Q^{r}\right\}=0$

Example 5.5. Let $Q^{i}=R^{1}, s^{i}\left(x^{i}\right)=x^{i}$ and $m^{1}\left(d x^{1}\right)=$ constant $\times d x^{1}$ (i.e. $\boldsymbol{X}^{1}$ may be considered as a one-dimensional Brownian motion). Then $U_{a^{2}}^{2}(x)=\int_{0}^{x} m^{2}\left(a^{2}-y, a^{2}+y\right) d y$ by the definition. Putting $p(a)$ $=P .\left(\sigma_{a}<\infty\right)$, for any fixed point $a=\left(a^{1}, a^{2}\right)$ we obtain Table 5.2. In particular if $m^{2}\left(d x^{2}\right)=$ constant $\times\left|x^{2}\right|^{\gamma} d x^{2}(\gamma>-1)$, then we have Table 5.3, where $A^{\circ}=\left\{\left(x^{1}, 0\right) ; x^{1} \in R^{1}\right\}$. In the case $\gamma=0$ these properties are well known, for $\boldsymbol{X}$ may be considered as a two-dimensional Brownian motion.

Example 5.6. Let $Q^{i}=R^{1}, s^{i}\left(x^{i}\right)=x^{i}$ and $m^{1}\left(d x^{1}\right)=$ constant $\times\left|x^{1}\right|^{\gamma}$ $d x^{1}(y>-1)$. Then for any fixed point $a=\left(a^{1}, a^{2}\right)$ we obtain the following table.

Table 5.4

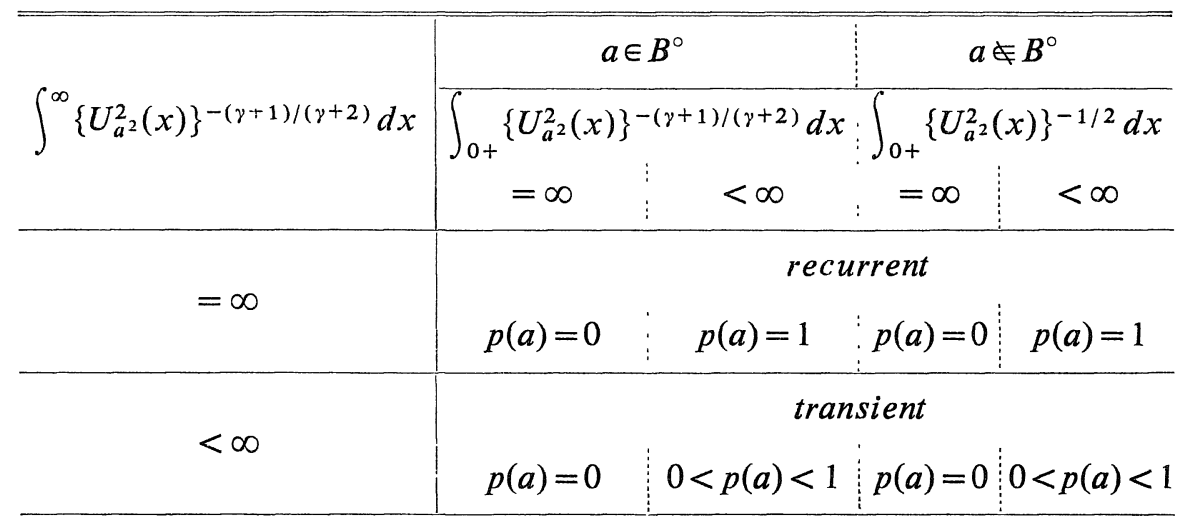

where $B^{\circ}=\left\{\left(0, x^{2}\right): x^{2} \in R^{1}\right\}$.

\section{References}

[1] Itô, K. and Mckean Jr., H. P., Diffusion processes and their sample paths, Springer, Berlin-Heidelberg-New York (1965).

[2] Kac, I. S., Integral characteristics of the growth of spectral functions for generalized second order boundary problems with conditions at a regular end, Izv. Akad. Nauk. SSSR Ser. Mat., 38 (1971), 154-184 (in Russian), Math. USSR-Izv., 5 (1971), 161-191 (Engl. transl.).

[3] — Generalization of an asymptotic formula of V. A. Marcenko for 
spectral functions of a second order boundary value problem, Izv. Akad. Nauk, SSSR Ser. Mat., 37 (1973), 422-436 (in Russian), Mat. USSR-Izv., 7 (1973), 422-436 (Engl. transl.).

[4] Kasahara, Y., Spectral theory of generalized second order differential operators and its applications to Markov processes, Japan J. Math., 1 (1975), 67-84.

[5] Tomisaki, M., Comparison theorems in generalized diffusion processes, Mem. Fac. Sci. Kyushu Univ., 30 (1976), 247-256. 